\title{
Methotrexate-associated lymphoproliferative disorders of T-cell phenotype: clinicopathological analysis of 28 cases
}

\author{
Akira Satou ${ }^{1}$ Tetsuya Tabata ${ }^{2}$ Hiroaki Miyoshi $\mathbb{1}^{3} \cdot$ Kei Kohno $^{4} \cdot$ Yuka Suzuki $^{4}$ - Daisuke Yamashita ${ }^{5}$. \\ Kazuyuki Shimada ${ }^{6} \cdot$ Tomonori Kawasaki $^{7} \cdot$ Yasuharu Sato $^{8} \cdot$ Tadashi Yoshino $^{2} \cdot$ Koichi Ohshima $^{3} \cdot$ Taishi Takahara $^{1}$. \\ Toyonori Tsuzuki ${ }^{1}{ }^{1} \cdot$ Shigeo Nakamura ${ }^{4}$
}

Received: 16 December 2018 / Revised: 26 February 2019 / Accepted: 27 February 2019 / Published online: 5 April 2019

(c) United States \& Canadian Academy of Pathology 2019

\begin{abstract}
Methotrexate-associated lymphoproliferative disorders are categorized as "other immunodeficiency-associated lymphoproliferative disorders in the WHO classification. Methotrexate-associated lymphoproliferative disorder is mainly a B-cell lymphoproliferative disorders or Hodgkin lymphoma type, whereas T-cell lymphoproliferative disorders are relatively rare (4-8\%). Only a small number of methotrexate-associated T-cell lymphoproliferative disorders have been detailed thus far. Because of the rarity, methotrexate-associated T-cell lymphoproliferative disorder has not been well studied and its clinicopathological characteristics are unknown. A total of 28 cases of methotrexate-associated T-cell lymphoproliferative disorders were retrospectively analyzed. Histologically and immunohistochemically, they were divided into three main types: angioimmunoblastic T-cell lymphoma $(n=19)$, peripheral T-cell lymphoma, NOS $(n=6)$, and CD8 ${ }^{+}$cytotoxic Tcell lymphoma $(n=3)$. Among the 28 cases, only one $\mathrm{CD}^{+}$cytotoxic T-cell lymphoma case was Epstein-Barr viruspositive. The other 27 cases were negative for Epstein-Barr virus on tumor cells, but scattered Epstein-Barr virus-infected Bcells were detected in 24 cases (89\%), implying the reactivation of Epstein-Barr virus caused by immunodeficient status of the patients. After the diagnosis of methotrexate-associated T-cell lymphoproliferative disorder, methotrexate was immediately withdrawn in 26 cases. Twenty (77\%) cases presented with spontaneous regression. Compared to methotrexateassociated B-cell lymphoproliferative disorder, patients with methotrexate-associated T-cell lymphoproliferative disorder had a significantly higher proportion of males $(p=0.035)$ and presence of B-symptoms $(p=0.036)$, and lower proportion of Epstein-Barr virus ${ }^{+}$tumor cells $(p<0.001)$. Although the difference was not significant, the methotrexate-associated T-cell lymphoproliferative disorder also had more frequent spontaneous regression $(p=0.061)$. In conclusion, methotrexateassociated T-cell lymphoproliferative disorder was divided into three main types: angioimmunoblastic T-cell lymphoma, peripheral T-cell lymphoma, NOS, and $\mathrm{CD} 8^{+}$cytotoxic T-cell lymphoma. Angioimmunoblastic T-cell lymphoma was the most common type. Methotrexate-associated T-cell lymphoproliferative disorder was characterized by a high rate of spontaneous regression after methotrexate cessation. Epstein-Barr virus positivity was relatively rare in methotrexateassociated T-cell lymphoproliferative disorder, significantly less frequent than methotrexate-associated B-cell lymphoproliferative disorder, suggesting different pathogenesis.
\end{abstract}

This work was presented at the USCAP Annual Meeting 2019 in National Harbor.

Supplementary information The online version of this article (https:// doi.org/10.1038/s41379-019-0264-2) contains supplementary material, which is available to authorized users.

Akira Satou

satoakira@aichi-med-u.ac.jp

Extended author information available on the last page of the article.

\section{Introduction}

Methotrexate is a folate analog cytoreductive drug commonly used for autoimmune disorders. Methotrexate suppresses the autoimmune state of rheumatoid arthritis patients and has an excellent inhibitory effect against articular destruction. Consequently, methotrexate is currently used as a first-line treatment in rheumatoid arthritis [1]. However, the risk of lymphoproliferative disorders is increased in patients treated with methotrexate [2]. In the recent WHO classification, methotrexate-associated 
lymphoproliferative disorders are categorized as "other immunodeficiency-associated lymphoproliferative disorders" [3]. The immunosuppressive state induced by methotrexate is considered to be a cause of methotrexateassociated lymphoproliferative disorders, but the mechanism of its development has not been well elucidated. In addition, patients with rheumatoid arthritis develop lymphoproliferative disorders 2.0 to 5.5 times more often than the general population [4-6]. The hyperimmune state of rheumatoid arthritis may contribute to the tumorigenesis of lymphoproliferative disorders. Therefore, how methotrexate influences the development of lymphoproliferative disorder remains controversial. On the other hand, a significant proportion of patients with methotrexate-associated lymphoproliferative disorders, particularly Epstein-Barr virus ${ }^{+}$ cases, have presented with spontaneous regression after methotrexate cessation [7-11]. This phenomenon is a characteristic of methotrexate-associated lymphoproliferative disorder and regarded as strong evidence for a potential tumorigenic role of methotrexate.

According to the WHO classification and previous large studies, methotrexate-associated lymphoproliferative disorder is mainly a B-cell lymphoproliferative disorder or Hodgkin lymphoma type, whereas T-cell lymphoproliferative disorders are relatively rare (4-8\%) [3, 7-10]. Only a small number of methotrexate-associated T-cell lymphoproliferative disorders have been detailed thus far: five cases of angioimmunoblastic T-cell lymphoma, four cases of Epstein-Barr virus ${ }^{+} \mathrm{CD} 8^{+}$T-cell lymphoproliferative disorders, one case of Epstein-Barr virus ${ }^{-} \mathrm{CD}^{+}{ }^{+} \mathrm{T}$-cell lymphoproliferative disorder, and one case of adult T-cell leukemia/lymphoma [7, 12-19].

Because of the rarity, methotrexate-associated lymphoproliferative disorder of T-cell phenotype has not been well studied and its clinicopathological characteristics are unknown. To further characterize methotrexate-associated T-cell lymphoproliferative disorder, we documented the clinicopathological characteristics of 28 cases of methotrexate-associated T-cell lymphoproliferative disorder and compared them to methotrexate-associated B-cell lymphoproliferative disorder and angioimmunoblastic Tcell lymphoma.

\section{Materials and methods}

\section{Patient samples}

A total of 28 cases of methotrexate-associated T-cell lymphoproliferative disorder diagnosed between 2008 and 2017 at five collaborating institutions were analyzed. We defined methotrexate-associated lymphoproliferative disorder as a lymphoproliferative disorder that developed in patients receiving methotrexate at the time of diagnosis. Cases were diagnosed according to the 2017 WHO classification based on morphology, immunophenotyping, and clinical information. All cases were independently reviewed by two pathologists (authors A.S. and S.N.) to confirm the diagnosis and immunophenotype. All of the cases had an Epstein-Barr virus past-infection pattern in a serum antibody test. No cases were serum-positive for HTLV-1 or human immunodeficiency virus antibody.

The control groups consisted of 33 patients consecutively diagnosed with methotrexate-associated B-cell lymphoproliferative disorder (14 diffuse large B-cell lymphoma and 19 polymorphic type arising in rheumatoid arthritis patients) and 213 patients with angioimmunoblastic T-cell lymphoma. The 33 cases of methotrexate-associated B-cell lymphoproliferative disorder were diagnosed between 2008 and 2017 in Nagoya University Hospital. Patients diagnosed with Epstein-Barr virus ${ }^{+}$mucocutaneous ulcer were not included in the methotrexate-associated B-cell lymphoproliferative disorder group. The angioimmunoblastic $\mathrm{T}$-cell lymphoma group had been examined in a previous study [20].

Clinical, laboratory, and follow-up data were obtained from the patient's medical records at each institution. Involved sites were usually examined by biopsy or radiographic evaluation (e.g., computed tomography and positron emission tomography). The institutional review board of Nagoya University and Aichi Medical University Hospital approved the study protocol.

\section{Histological and immunohistochemical staining}

Tissue samples were fixed in $10 \%$ formalin, embedded in paraffin, and 4- $\mu$ m-thick sections stained with hematoxylin and eosin. Immunoperoxidase studies were performed on formalin-fixed paraffin-embedded sections. The following monoclonal antibodies were used: CD3, CD8, CD30 (DAKO, Santa Fe, CA), CD4, perforin (Novocastra Laboratories, Newcastle, UK), PD1 (Abcam, Cambridge, MA), CXCL13 (R\&D systems, Minneapolis, MA), TIA-1 (Coulter Immunology, Hialeah, FL), granzymeB (Mososan, Uden, the Netherlands), CD56 (eBioscience, San Diego, CA), and PD-L1 (SP142; Spring Bioscience, Pleasanton, CA, USA). These antibodies were used after antigen retrieval following heating in microwave oven. With the exception of PD-L1, tissue samples were considered positive if more than $30 \%$ of the tumor cells were positive. Tumor cells were considered positive for PD-L1 when $\geq 5 \%$ of the neoplastic lymphoid cells demonstrated moderate or strong membrane staining with a PD-L1-specific antibody. A case was considered positive for PD-L1 in the microenvironment when, among the total tissue cellularity, $\geq 20 \%$ comprised nonmalignant cells with 
moderate or strong membrane or cytoplasmic PD-L1specific staining.

\section{In situ hybridization study}

The presence of Epstein-Barr virus-encoded small RNA was examined by in situ hybridization using Epstein-Barr virus-encoded small nuclear early region oligonucleotides on formalin-fixed, paraffin-embedded sections as described previously [21].

\section{TCRY and IgH PCR study}

DNA was extracted from formalin-fixed tissue, and PCR analysis of the TCR $\gamma$ and IgH genes carried out using the BIOMED2 protocol as described previously [22].

\section{Statistical analysis}

Patient survival data were analyzed by the Kaplan-Meier method. Differences in survival were tested by the log-rank test. Overall survival was calculated from the diagnosis date to the date of death or last date of follow-up. Diseasespecific survival was measured from the date of diagnosis to the date of disease-specific death or the last date of followup. Progression-free survival was calculated from the diagnosis date to the first date of disease progression, relapse, or death as a result of any cause or the last date of follow-up. All statistical analyses were performed using the STATA software package version 12 (STATA Corporation, College Station, TX, USA).

\section{Results}

\section{Clinical characteristics of methotrexate-associated T-cell lymphoproliferative disorder}

The clinical features of the methotrexate-associated T-cell lymphoproliferative disorder cases are summarized in Table 1 and Supplementalary Table 1. The 15 men and 13 women had a median age of 70 years (range, 56-85). All patients were treated with methotrexate for rheumatoid arthritis at the onset of methotrexate-associated T-cell lymphoproliferative disorder. The median duration of methotrexate use was 3 years (range, 0.5-21). At the time of diagnosis, 21 patients (78\%) showed stage III/IV disease, $15(58 \%)$ were categorized as high-intermediate/high risk group according to International Prognostic Index, and none of them had bulky mass. Laboratory data revealed elevated level of lactate dehydrogenase in 15 patients (54\%) and soluble interleukin-2 receptor in all 17 patients for whom data were available.
Table 1 Clinical features of methotrexate-associated T-cell lymphoproliferative disorder (MTX T-LPD) compared to methotrexate-associated B-cell lymphoproliferative disorder (MTX B-LPD)

\begin{tabular}{llll}
\hline Variable & $\begin{array}{l}\text { MTX T-LPD } \\
(n=28)\end{array}$ & $\begin{array}{l}\text { MTX B-LPD } \\
(n=33)\end{array}$ & $P$ \\
\hline Sex (M/F) & $15 / 13$ & $10 / 23$ & 0.035 \\
Age (median[range]) & $70(56-85)$ & $68(47-82)$ & 0.27 \\
$\begin{array}{l}\text { MTX duration }(y) \\
\text { (median[range]) }\end{array}$ & $3(0.5-21)$ & $6(0.3-16)$ & 0.35 \\
PS $>1$ & $7 / 28(25 \%)$ & $11 / 30(37 \%)$ & 0.34 \\
Stage III/IV & $21 / 26(81 \%)$ & $26 / 33(79 \%)$ & 0.92 \\
B symptom & $15 / 28(54 \%)$ & $9 / 33(27 \%)$ & 0.036 \\
Bulky mass & $0 / 28(0 \%)$ & $1 / 31(1.7 \%)$ & - \\
Extranodal site $>1$ & $7 / 27(26 \%)$ & $9 / 33(27 \%)$ & 0.91 \\
LDH>normal & $15 / 28(54 \%)$ & $24 / 32(75 \%)$ & 0.083 \\
IPI HI/H & $15 / 26(58 \%)$ & $20 / 29(69 \%)$ & 0.56 \\
EBER & $1 / 28(3.6 \%)$ & $20 / 33(61 \%)$ & $<0.001$ \\
SR & $20 / 26(77 \%)$ & $17 / 32(53 \%)$ & 0.061 \\
\hline
\end{tabular}

EBER Epstein-Barr virus-encoded small RNA, $F$ female, $H$ high, $H I$ high-intermediate, IPI international prognostic index, $M$ male, MTX methotrexate, $L D H$ lactate dehydrogenase, $L P D$ lymphoproliferative disorder, $P S$, performance status, $S R$ spontaneous regression, $y$ year

The disease sites and biopsy sites for each case are given in Supplementary Table 1. Briefly, at the time of diagnosis, 27 patients (96\%) had lymphadenopathy, including 11 who also had extranodal involvement, and 1 patient (3.6\%) had localized cutaneous disease. The most frequent sites of extranodal involvement were the skin $(n=3)$, spleen $(n=3)$, liver $(n=2)$, and bone marrow $(n=2)$.

Compared with methotrexate-associated B-cell lymphoproliferative disorder, patients with methotrexate-associated T-cell lymphoproliferative disorder had a significantly higher proportion of males $(p=0.035)$ and presence of Bsymptoms $(p=0.036)$, and lower proportion of EpsteinBarr virus $^{+}$tumor cells $(p<0.001)$. Although the difference was not significant, the methotrexate-associated $\mathrm{T}$-cell lymphoproliferative disorder group also had more frequent spontaneous regression $(p=0.061)$.

\section{Histological, immunohistochemical, and molecular features}

Histological, immunohistochemical, and molecular features are summarized in Supplementary Table 2. Twenty-eight cases of methotrexate-associated T-cell lymphoproliferative disorders were divided into three main types: Angioimmunoblastic T-cell lymphoma $(n=19)$, peripheral T-cell lymphoma, NOS $(n=6)$, and CD8 ${ }^{+}$cytotoxic T-cell lymphoma $(n=3)$.

Histologically, angioimmunoblastic T-cell lymphoma type exhibited diffuse and polymorphous infiltration of 
Fig. 1 Histological and immunohistochemical features of angioimmunoblastic T-cell lymphoma-type methotrexateassociated T-cell

lymphoproliferative disorder.

The angioimmunoblastic T-cell lymphoma type had diffuse and polymorphous infiltration of small-sized to medium-sized lymphocytes intermingled with plasma cells, histiocytes, and eosinophils, accompanied by proliferation of high endothelial venules. Small-sized to mediumsized lymphocytes were

characterized by clear cytoplasm (a: $\mathrm{HE} \times 200$, b: HE $\times 400$ ). Immunohistochemically, the tumor cells were positive for PD1 (c: PD1 ×200). Six Aangioimmunoblastic T-cell lymphoma cases expressed PD-L1 on microenvironment immune cells $(\mathbf{d}$ : PD-L1 ×200). Scattered Epstein-Barr virus EBV-infected B cells were detected in the background (e: Epstein-Barr virus-encoded small RNA [EBER] ×200), including Epstein-Barr virusinfected B cells mimicking Hodgkin-Reed-Sternberg cells (f: $\mathrm{HE} \times 400$, G: EBER ×400)
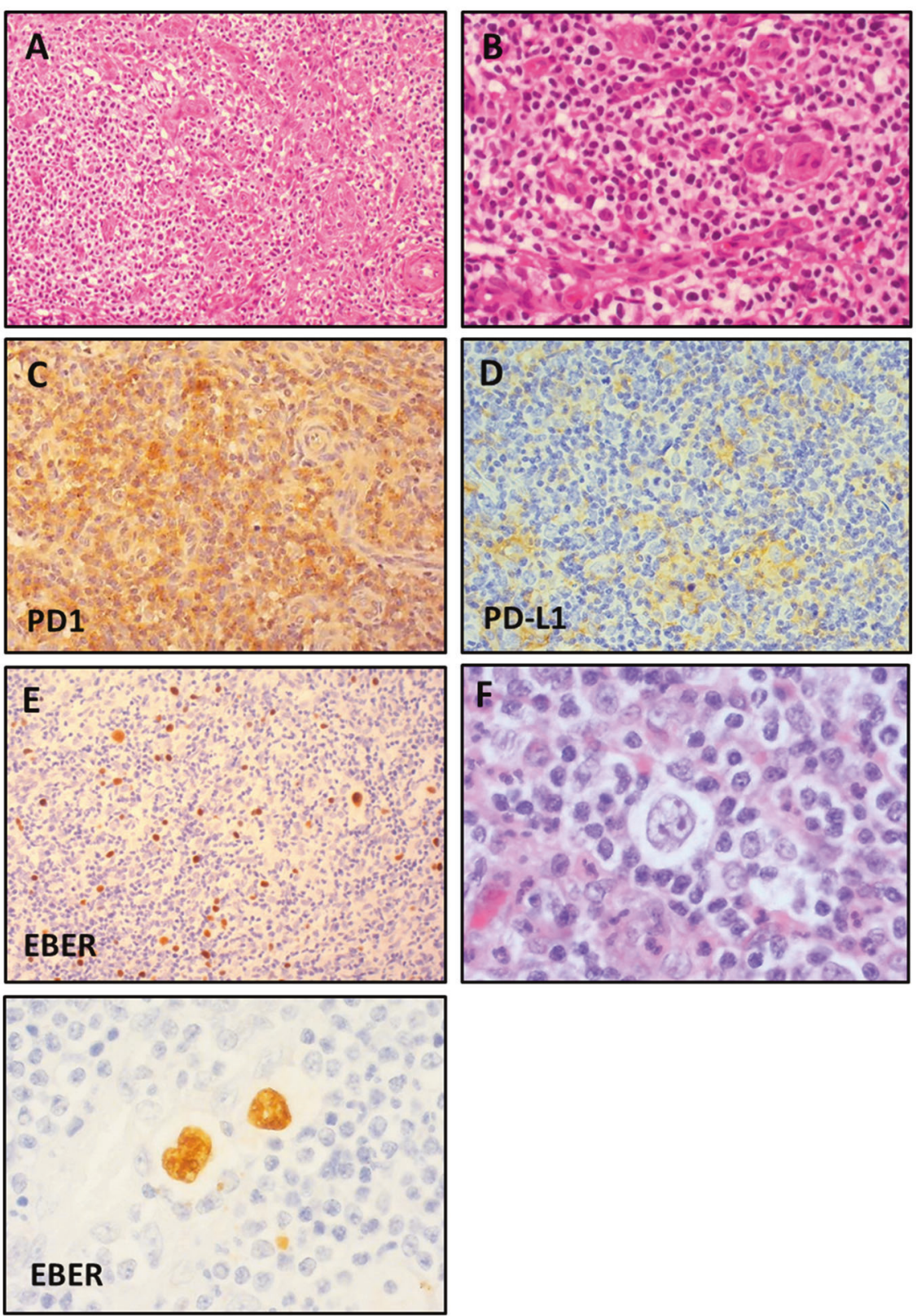

small-sized to medium-sized lymphocytes intermingled with plasma cells, histiocytes, and eosinophils, accompanied by proliferation of high endothelial venules. Smallsized to medium-sized lymphocytes were characterized by clear cytoplasm (Fig. 1a, b). Immunohistochemically, all tested cases were positive for CD3, CD4, and PD1 (Fig. 1c) and/or CXCL13 and negative for CD8.

Peripheral T-cell lymphoma, NOS type exhibited diffuse infiltration of medium- to large-sized atypical lymphocytes (Fig. 2a, b). Among the six peripheral T-cell lymphoma, NOS cases, case \#25 had a focal lesion on the skin of the face; this case had swelling of the facial skin and tumor cells infiltrating the subcutaneous tissue and dermis. Immunohistochemically, in all six cases, tumor cells were positive for CD3 (Fig. 2c). Four cases were positive for CD4 (Fig. 2d) and negative for $\mathrm{CD} 8$, one case was negative for both CD4 and CD8, and one case demonstrated focal positivity for both CD4 and CD8. The case with focal positivity for CD8 was negative for cytotoxic molecule.

Regarding the $\mathrm{CD} 8^{+}$cytotoxic T-cell lymphoma type, all three cases had both nodal and extranodal lesions. Case \#26 was diagnosed by biopsy of the nodal site, and the other two cases by extranodal sites (case \#27, bone marrow; and case \#28, liver). Histologically, case \#26 exhibited diffuse infiltration of medium-sized atypical lymphocytes. The other two cases were characterized by infiltration of mediumsized atypical lymphocytes (Fig. 2e). Immunohistochemically, all three cases were positive for CD3, CD8 (Fig. 2f), 
Fig. 2 Histological and immunohistochemical features of peripheral T-cell lymphoma, NOS-type and Epstein-Barr virus ${ }^{+} \mathrm{CD}^{+}$cytotoxic T-cell lymphoma-type methotrexateassociated T-cell lymphoproliferative disorder. The peripheral T-cell lymphoma, NOS type exhibited diffuse infiltration of mediumsized to large-sized atypical lymphocytes (a: $\mathrm{HE} \times 100$, b: HE $\times 400)$. The tumor cells were positive for CD3 (c: CD3 $\times 200)$. The majority of the cases were positive for CD4 (d: CD4 $\times 100)$. The Epstein-Barr virus ${ }^{+} \mathrm{CD} 8^{+}$ cytotoxic T-cell lymphoma type was characterized by infiltration of medium-sized atypical lymphocytes (e: HE $\times 400)$. Immunohistochemically, tumor cells were positive for $\mathrm{CD} 8$ (f: HE $\times 400)$, TIA-1 (g: HE $\times 400)$, and Epstein-Barr virus encoded small RNA (EBER) (h: EBER $\times 400$ )
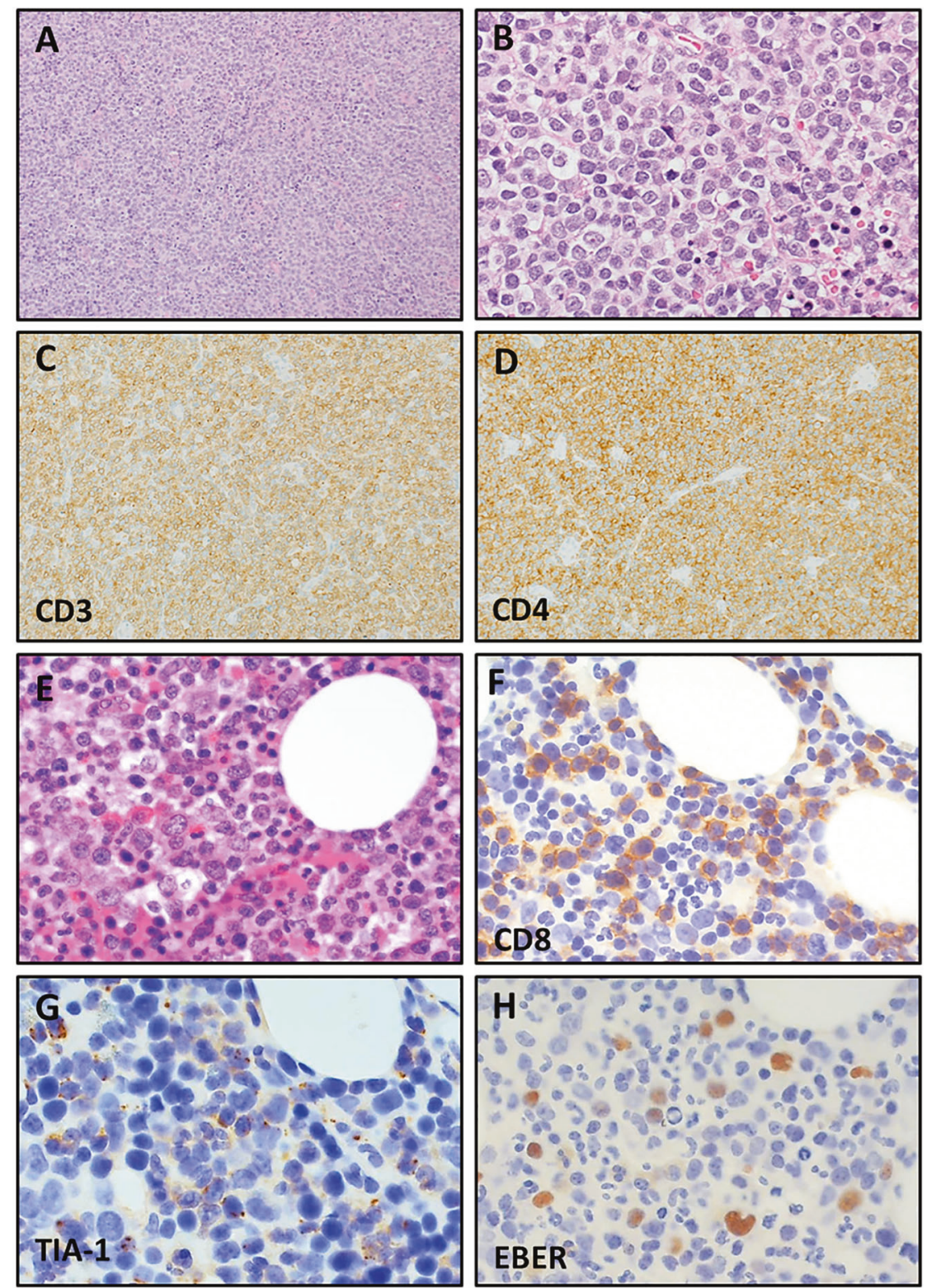

and cytotoxic molecule (Fig. 2g), but negative for CD4 and CD56. Notably, the tumor cells in case \#27 were infected with Epstein-Barr virus (Fig. 2h); therefore, some may assert that this case should be diagnosed as NK/T cell lymphoma. However, this case exhibited clonal rearrangement of the TCR $\gamma$ gene and negativity for CD56, suggesting a T-cell lineage of the tumor. Thus, we preferred the diagnosis of Epstein-Barr virus ${ }^{+} \mathrm{CD}^{+}$cytotoxic $\mathrm{T}$-cell lymphoma.

PD-L1 expression was assessed in 14 cases (10 angioimmunoblastic T-cell lymphoma, $3 \mathrm{CD}^{+}$cytotoxic T-cell lymphoma, and 1 peripheral T-cell lymphoma, NOS type). Tumor cells were negative for PD-L1 in all 14 cases. Nine (6 angioimmunoblastic T-cell lymphoma, $2 \mathrm{CD}^{+}$ cytotoxic T-cell lymphoma, and 1 peripheral $\mathrm{T}$-cell lymphoma, NOS type) of the 14 cases expressed PD-L1 on microenvironment immune cells (Fig. 1d).

Interestingly, two cases (case \#7 and \#8) exhibited metachronous or synchronous coexistence of angioimmunoblastic T-cell lymphoma and B-cell lymphoproliferative disorder. Case \#7 initially had an angioimmunoblastic Tcell lymphoma lesion in the lymph node. When the patient relapsed, the lymph node exhibited diffuse large B-cell lymphoma pathology. In contrast, at the time of diagnosis, case \#8 had both a nodal lesion with angioimmunoblastic Tcell lymphoma features (Fig. 3a-c) and a cutaneous lesion diagnosed as Epstein-Barr virus ${ }^{+}$monomorphic B-cell lymphoproliferative disorder (Fig. 3d-f).

$T C R \gamma$ and $I g H$ PCR analysis was performed in 11 and 10 cases of angioimmunoblastic T-cell lymphoma type and in 

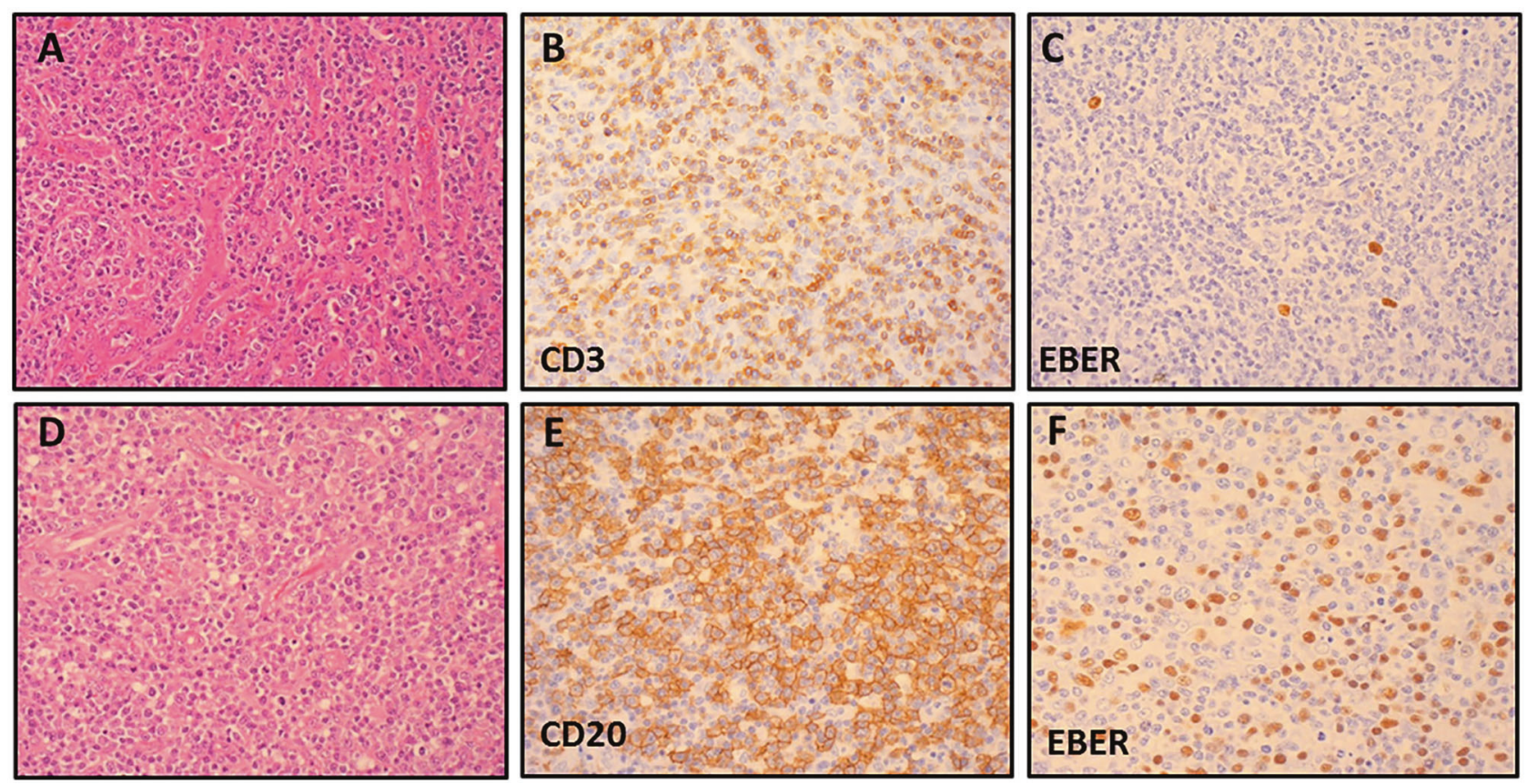

Fig. 3 Histological and immunohistochemical features of case \#8, which exhibited synchronous coexistence of angioimmunoblastic $\mathrm{T}$ cell lymphoma (nodal lesion) and Epstein-Barr virus-positive monomorphic B-cell lymphoproliferative disorder (cutaneous lesion). The nodal lesion showed histological features of angioimmunoblastic Tcell lymphoma (a: $\mathrm{HE} \times 200)$. The tumor cells were positive for CD3 (b: CD3 ×200). Scattered Epstein-Barr virus-infected B cells were detected in the background (c: Epstein-Barr virus-encoded small RNA [EBER] $\times 200$ ). Cutaneous lesion had diffuse infiltration of mediumsized to large-sized atypical lymphocytes $(\mathbf{d}$ : HE $\times 200)$ positive for CD20 (e: CD20 ×200) and EBER $(\mathbf{f : ~ E B E R ~} \times 200)$
3 and 2 cases of the other two types. Regarding angioimmunoblastic T-cell lymphoma type, a clonal $T C R \gamma$ rearrangement was detected in $9(82 \%)$ cases and $\operatorname{IgH}$ rearrangement in $3(30 \%)$ cases. One $(33 \%)$ case of peripheral T-cell lymphoma, NOS and $\mathrm{CD}^{+}$cytotoxic T-cell lymphoma type showed clonal $T C R \gamma$ rearrangement and none of them showed clonal $\operatorname{IgH}$ rearrangement. Rearrangement of both $T C R \gamma$ and $\operatorname{IgH}$ was found in two cases of angioummunoblastic T-cell lymphoma type. No rearrangements of these genes were found in one each of angioimmunoblastic T-cell lymphoma, peripheral T-cell lymphoma, NOS and $\mathrm{CD}^{+}$cytotoxic T-cell lymphoma type. Rearrangement of only $\mathrm{IgH}$ was found in one case of angioimmunoblastic T-cell lymphoma type. The other eight cases (7 angioimmunoblastic T-cell lymphoma and $1 \mathrm{CD} 8^{+}$ cytotoxic T-cell lymphoma type) had rearrangement of $T C R \gamma$ only.

\section{Epstein-Barr virus infection}

Epstein-Barr virus-encoded small RNA by in situ hybridization which was used to analyze all cases. As mentioned above, only one case expressed Epstein-Barr virus-encoded small RNA on tumor cells. Tumor cells from the other 27 cases were negative for Epstein-Barr virus-encoded small RNA, but scattered Epstein-Barr virus-infected B cells were detected in 24 cases (89\%), implying the reactivation of Epstein-Barr virus caused by the immunodeficient status of the patients (Fig. 1d). In more detail, 17 cases (89\%) of angioimmunoblastic T-cell lymphoma, 5 (83\%) of peripheral T-cell lymphoma, NOS and $2(100 \%)$ of $\mathrm{CD}^{+}$ cytotoxic T-cell lymphoma type contained Epstein-Barr virus-infected B cells. Notably, four cases (3 angioimmunoblastic T-cell lymphoma and one peripheral T-cell lymphoma, NOS) were accompanied by Epstein-Barr virusinfected B cells mimicking Hodgkin-Reed-Sternberg cells (Fig. 1f, g).

\section{Therapy and prognosis}

After the diagnosis of methotrexate-associated T-cell lymphoproliferative disorder, methotrexate was immediately withdrawn in 26 cases. Twenty $(77 \%)$ cases presented with spontaneous regression after cessation of methotrexate. Nine patients received cytotoxic chemotherapy as the initial treatment; five were treated with CHOP (combination of cyclophosphamide, adriamycin, vincristine and prednisone), two with R-CHOP (CHOP with rituximab), one with THP-COP (combination of pirarubicin, cyclophosphamide, vincristine and prednisolone), and one with sobuzoxane and etoposide. Among the nine patients, two received cytotoxic chemotherapy even though they showed 
A Disease-specific survival curve

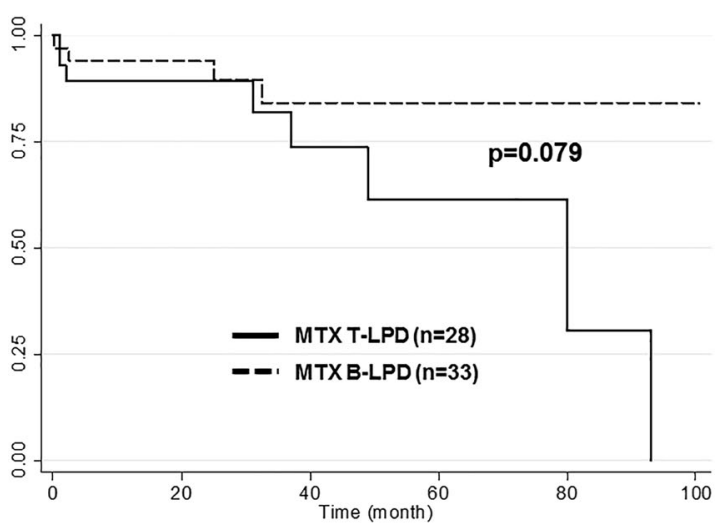

Fig. 4 Disease-specific survival curve and progression-free survival curves for methotrexate-associated T-cell lymphoproliferative disorder compared to methotrexate-associated B-cell lymphoproliferative disorder (MTX B-LPD). Patients with methotrexate-associated T-cell

spontaneous regression after methotrexate cessation. Eventually, 24 cases achieved complete remission and two cases partial response. Four of the 26 patients had relapse or progression. We compared the disease-specific survival and progression-free survival curves for methotrexateassociated T-cell lymphoproliferative disorder to those of methotrexate-associated B-cell lymphoproliferative disorder. Patients with methotrexate-associated T-cell lymphoproliferative disorder tended to have inferior survival curves (disease-specific survival, $p=0.079$ and progression-free survival $p=0.095$ ) (Fig. 4). We further divided methotrexate-associated B-cell lymphoproliferative disorder cases into diffuse large B-cell lymphoma and polymorphic types and compared the disease-specific survival and progression-free survival curves for each type to those of methotrexate-associated T-cell lymphoproliferative disorder. Compared with both diffuse large B-cell lymphoma and polymorphic types, patients with methotrexateassociated T-cell lymphoproliferative disorder had inferior disease-specific survival $(p=0.18$ and $p=0.15$, respectively) and progression-free survival $(p=0.21$ and $p=$ 0.22 , respectively) curves, though the differences were not significant. Among the 33 cases of methotrexate-associated B-cell lymphoproliferative disorder, 15 patients received chemotherapy as an initial treatment: R-CHOP $(n=7), \mathrm{R}$ only $(n=7)$, and CHOP $(n=1)$.

\section{Comparison of methotrexate-associated T-cell lymphoproliferative disorder of angioimmunoblastic T-cell type and angioimmunoblastic T-cell lymphoma}

As the majority of methotrexate-associated T-cell lymphoproliferative disorder had an angioimmunoblastic T-cell lymphoma pattern, we compared the clinical characteristics

\section{B Progression-free survival curve}

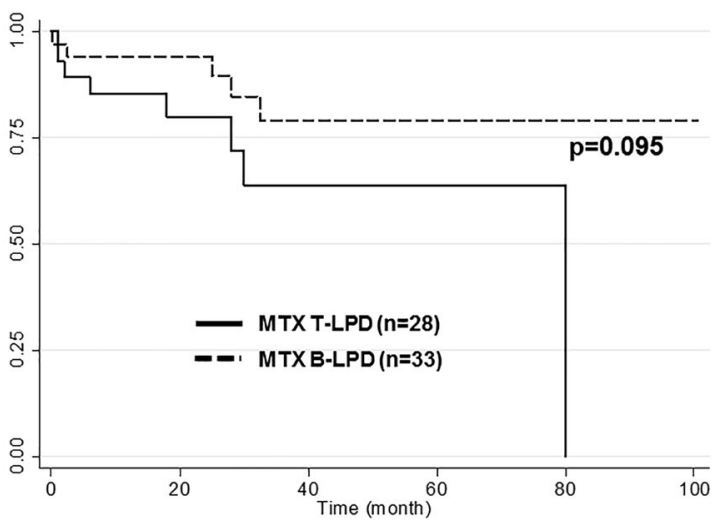

lymphoproliferative disorder (MTX T-LPD) tended to have inferior survival in both disease-specific survival (a) and progression-free survival (b) ( $p=0.079$ and 0.095 , respectively)

Table 2 Clinical features of methotrexate-associated T-cell lymphoproliferative disorder of angioimmunoblastic T-cell lymphoma type (MTX-AITL) compared to angioimmunoblastic T-cell lymphoma (AITL)

\begin{tabular}{llll}
\hline Variable & $\begin{array}{l}\text { MTX-AITL } \\
(n=19)\end{array}$ & $\begin{array}{l}\text { AITL } \\
(n=213)\end{array}$ & $P$ \\
\hline Sex (M/F) & $13 / 6$ & $137 / 76$ & 0.81 \\
Age (median[range]) & $72(57-85)$ & $67(34-91)$ & 0.0037 \\
PS $>1$ & $5 / 19(26 \%)$ & $75 / 209(36 \%)$ & 0.46 \\
Stage III/IV & $16 / 18(89 \%)$ & $189 / 211(89 \%)$ & - \\
B symptom & $11 / 19(58 \%)$ & $123 / 205(60 \%)$ & - \\
Extranodal site $>1$ & $4 / 18(22 \%)$ & $45 / 200(23 \%)$ & - \\
LDH > normal & $10 / 19(53 \%)$ & $157 / 209(75 \%)$ & 0.054 \\
IPI HI/H & $11 / 17(65 \%)$ & $138 / 199(69 \%)$ & 0.79 \\
EBV in background & $17 / 19(89 \%)$ & $55 / 166(67 \%)$ & 0.064 \\
\hline
\end{tabular}

AITL angioimmunoblastic T-cell lymphoma, $E B V$ Epstein-Barr virus, $F$ female, $H$ high, $H I$ high-intermediate, $I P I$ international prognostic index, $L D H$ lactate dehydrogenase, $M$ male, MTX-AITL MTXassociated lymphoproliferative disorder of AITL type, $P S$ performance status

and prognosis of methotrexate-associated T-cell lymphoproliferative disorder of angioimmunoblastic T-cell type and angioimmunoblastic T-cell lymphoma patients (Table 2). Compared wth the angioimmunoblastic T-cell lymphoma group, the methotrexate-associated T-cell lymphoproliferative disorder of angioimmunoblastic T-cell lymphoma type had a significantly higher age distribution $(p=0.0037)$. In addition, methotrexate-associated T-cell lymphoproliferative disorder of angioimmunoblastic T-cell lymphoma type tended to have scattered Epstein-Barr virus $^{+}$cells in the background more frequently $(p=$ $0.064)$ and less likely to have an elevated lactate dehydrogenase level $(p=0.054)$. Overall survival and progression-free survival curves for the two groups were 


\section{A Overall survival curve}

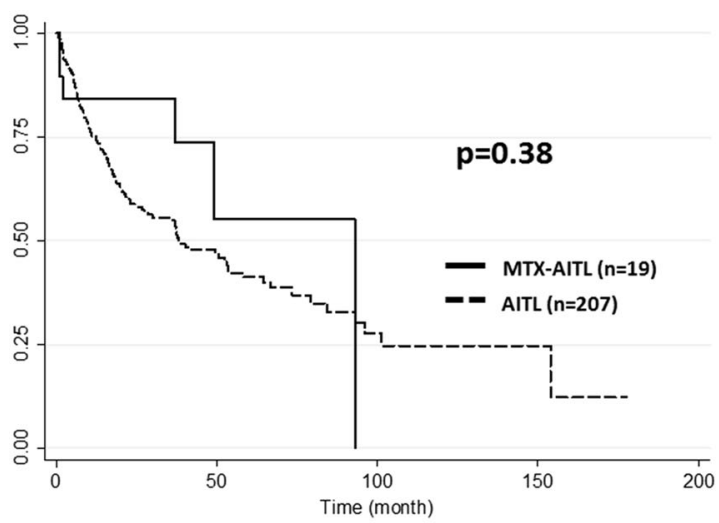

\section{Overall survival curve ( $\geqq 60$ years)}

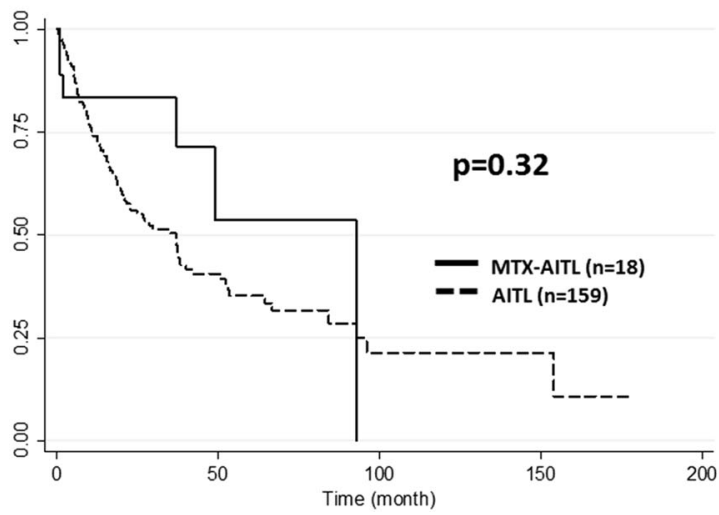

Fig. 5 Overall survival and disease-specific survival curves for methotrexate-associated T-cell lymphoproliferative disorder of angioimmunoblastic T-cell lymphoma type compared to angioimmunoblastic T-cell lymphoma. Patients with methotrexate-associated T-cell lymphoproliferative disorder of angioimmunoblastic T-cell lymphoma type (MTX-AITL) had superior overall survival curve

compared. Patients with methotrexate-associated T-cell lymphoproliferative disorder of angioimmunoblastic T-cell type had superior overall survival and progression-free survival curves compared with angioimmunoblastic T-cell lymphoma, though the differences were not significant (Fig. 5a, b). Considering the fact that the methotrexateassociated T-cell lymphoproliferative disorder of angioimmunoblastic T-cell type had a significantly higher age distribution, we also compared the overall survival and progression-free survival curves for the patients of the two groups who are 60 years or older. The survival curves showed the same trend (Fig. $5 \mathrm{c}, \mathrm{d}$ ).

\section{Discussion}

In the present study, we reported 28 cases of methotrexateassociated T-cell lymphoproliferative disorder. Previously, 11 cases of methotrexate-associated T-cell
B Progression-free survival curve

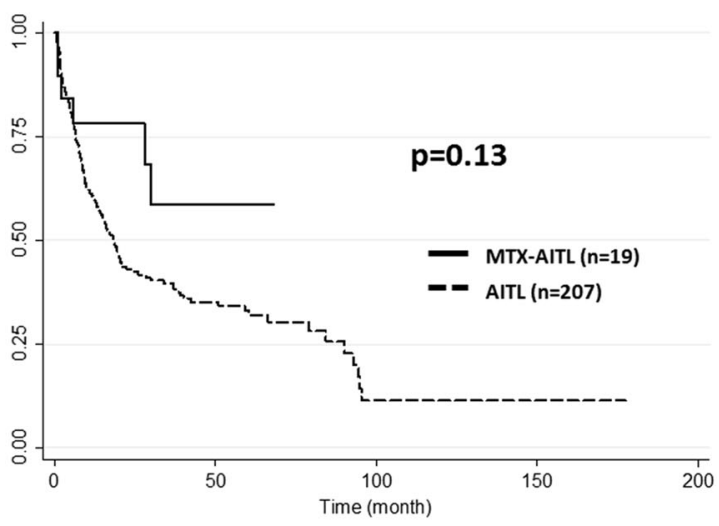

D Progression-free survival curve ( $\geqq 60$ years)

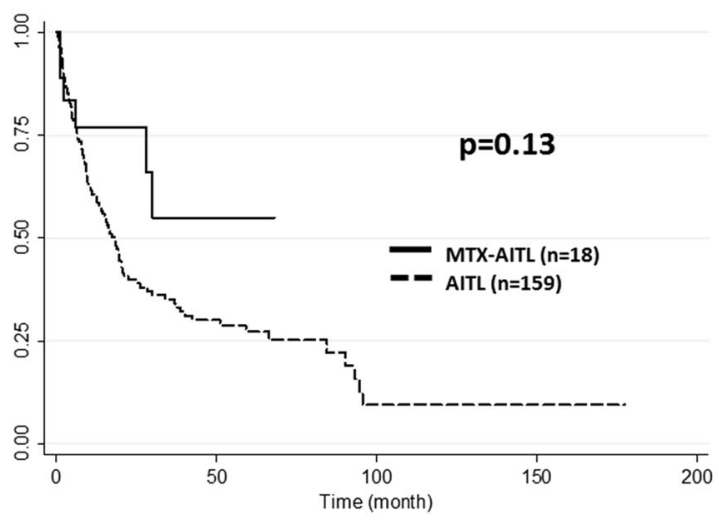

and progression-free survival curve, though the differences from angioimmunoblastic T-cell lymphoma (AITL) were not significant (a, b). Overall survival and progression-free survival curves for the patients of the two groups who are 60 years or older were also compared. The survival curves showed the same trend $(\mathbf{c}, \mathbf{d})$

lymphoproliferative disorder had been reported in the English literature with detailed descriptions: angioimmunoblastic T-cell lymphoma $(n=5)$, Epstein-Barr virus ${ }^{+}$ $\mathrm{CD}^{+}{ }^{+}$-cell lymphoproliferative disorder $(n=4)$, EpsteinBarr virus $\mathrm{CD}^{+}{ }^{+}$T-cell lymphoproliferative disorder $(n=$ $1)$, and adult T-cell leukemia/lymphoma $(n=1)$ [7, 12-19]. Reviews of the reported cases of methotrexate-associated Tcell lymphoproliferative disorder are summarized in Table 3. The peripheral T-cell lymphoma, NOS type has not been reported in previous papers, and amgioimmunoblastic T-cell lymphoma and $\mathrm{CD}^{+}$T-cell lymphoproliferative disorder were the major types of methotrexate-associated Tcell lymphoproliferative disorder. The cases in which tumor cells were Epstein-Barr virus ${ }^{+}$were also limited to $\mathrm{CD} 8^{+} \mathrm{T}$ cell lymphoproliferative disorder cases. Among the five $\mathrm{CD}^{+}{ }^{+}$T-cell lymphoproliferative disorder cases, only one case was examined for cytotoxic positivity and was found to be positive. All 11 cases presented with spontaneous regression after discontinuation of methotrexate. Combined 


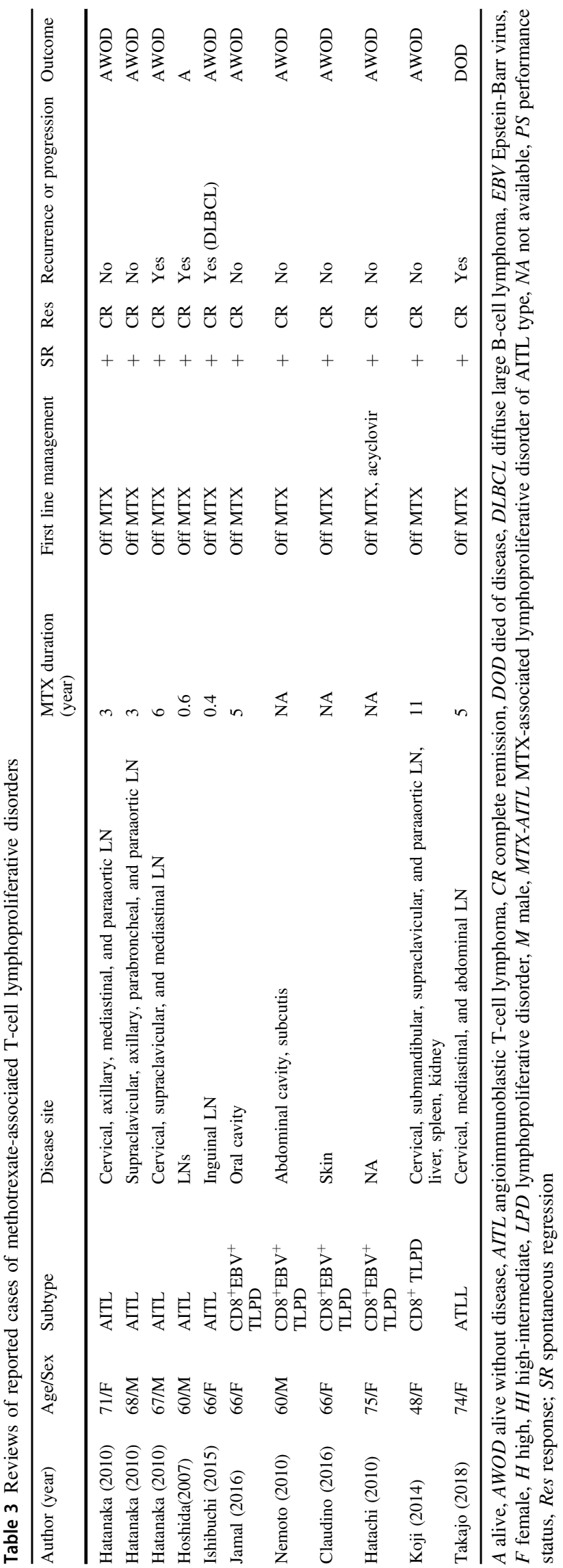

with our data, although some may have relapses later, spontaneous regression may be expected in the majority of methotrexate-associated T-cell lymphoproliferative disorder cases. Notably, all eight $\mathrm{CD}^{+}{ }^{-}$T-cell lymphoproliferative disorder cases achieved complete response after withdrawal of methotrexate and none of the cases experienced relapse. Therefore, none of the $\mathrm{CD} 8^{+} \mathrm{T}$-cell lymphoproliferative disorder patients required cytotoxic chemotherapy during their clinical course. According to previous reports, the rate of spontaneous regression was significantly higher in Epstein-Barr virus ${ }^{+}$methotrexate-associated lymphoproliferative disorder cases than Epstein-Barr virus ${ }^{-}$cases $[3,7,8]$. We consider that this is not applicable to methotrexate-associated T-cell lymphoproliferative disorder. As shown by our data, methotrexate-associated T-cell lymphoproliferative disorder cases have a high rate of spontaneous regression, even though most of them are Epstein-Barr virus ${ }^{-}$. A significant proportion of patients with methotrexate-associated B-cell lymphoproliferative disorder and Hodgkin lymphoma type present with spontaneous regression after methotrexate cessation. Thus, chemotherapy cannot be started immediately after the diagnosis of methotrexate-associated B-cell lymphoproliferative disorder and Hodgkin lymphoma type. Based on the findings of the present and previously reported cases, we conclude that the withdrawal of methotrexate should be done as the initial management after the diagnosis of methotrexateassociated T-cell lymphoproliferative disorder.

Interestingly, two of the current cases exhibited metachronous or synchronous coexistence of angioimmunoblastic T-cell lymphoma and B-cell lymphoproliferative disorder (diffuse large B-cell lymphoma and Epstein-Barr virus $^{+}$diffuse large B-cell lymphoma). Ishibuchi et al. also reported the case of methotrexate-associated T-cell lymphoproliferative disorder which showed metachronous coexistence of angioimmunoblastic T-cell lymphoma and Epstein-Barr virus $^{+}$diffuse large B-cell lymphoma (Table 3) [13]. Serial or simultaneous occurrence of angioimmunoblastic T-cell lymphoma and other lymphomas has been well documented. Attygalle et al. [23]. reported that 8 of 31 angioimmunoblastic T-cell lymphoma cases developed lymphoma (Epstein-Barr virus ${ }^{+}$diffuse large B-cell lymphoma, $n=5$; Epstein-Barr virus ${ }^{-}$diffuse large B-cell lymphoma, $n=1$; and Epstein-Barr virus ${ }^{+}$ Hodgkin lymphoma, $n=2$ ). Willenbrock et al. [24]. also documented 21 angioimmunoblastic T-cell lymphoma patients with lymphoma (Epstein-Barr virus ${ }^{+}$diffuse large B-cell lymphoma, $n=6$; Epstein-Barr virus ${ }^{-}$diffuse large B-cell lymphoma, $n=9$; diffuse large B-cell lymphoma, $n=1$; lymphoplasmacytic lymphoma, $n=2$; Epstein-Barr virus $^{+} \mathrm{CD} 30^{+}$lymphoma, $n=1$; and Epstein-Barr virus ${ }^{+}$ Hodgkin lymphoma, $n=2$ ) during their disease course. Angioimmunoblastic T-cell lymphoma usually contains 
B-cell blasts, which are often Epstein-Barr virus ${ }^{+}$, and clonal $\mathrm{IgH}$ rearrangements have been found in approximately $25-30 \%$ of angioimmunoblastic T-cell lymphoma cases [3]. These data suggest that the pathogenesis of other lymphomas that appear during the disease course of angioimmunoblastic T-cell lymphoma could be explained by clonal expansion of B cells [24]. Among the current 12 methotrexate-associated T-cell lymphoproliferative disorder cases analyzed by $\operatorname{IgH}$ PCR, clonal rearrangement was detected in $3(25 \%)$. Therefore, we assume that monoclonal $\mathrm{B}$ cells in methotrexate-associated T-cell lymphoproliferative disorder may also progress to B-cell lymphoproliferative disorder.

In our cohort, only one case of methotrexate-associated T-cell lymphoproliferative disorder had Epstein-Barr virus ${ }^{+}$ tumor cells, and methotrexate-associated T-cell lymphoproliferative disorder cases were characterized by a significantly lower ratio of Epstein-Barr virus positivity $(p<$ 0.001) compared with MTX-associated B-cell lymphoproliferative disorder. Regardless of cell lineage and EpsteinBarr virus positivity, the immunosuppressive state induced by methotrexate is considered as a common cause of methotrexate-associated lymphoproliferative disorder. Feng et al. [25]. suggested another potential cause of Epstein-Barr virus $^{+}$methotrexate-associated lymphoproliferative disorder. They reported that, in contrast to other causes of immunodeficiency, methotrexate may directly reactivate latent Epstein-Barr virus and contribute to the development of lymphoproliferative disorder. Therefore, considering the significant difference in Epstein-Barr virus positivity, we speculate that the pathogenesis may be different between the two groups.

Although only one case of methotrexate-associated Tcell lymphoproliferative disorder had Epstein-Barr virus ${ }^{+}$ tumor cells, scattered Epstein-Barr virus-infected B cells were detected in 24 of 27 Epstein-Barr virus ${ }^{-}$cases, implying the reactivation of Epstein-Barr virus caused by the immunodeficient status of the patients. We conclude that the function of Epstein-Barr virus-specific cytotoxic $\mathrm{T}$ lymphocytes was suppressed. This dysregulation in the immune surveillance for Epstein-Barr virus is assumed to result in the emergence of Epstein-Barr virus-infected B cells in the background [26]. In addition to the immunosuppression caused by methotrexate, considering the age of our cases (median 70 years; range, 56-85 years) and significantly higher age of onset of methotrexate-associated T-cell lymphoproliferative disorder of angioimmunoblastic T-cell lymphoma type compared with angioimmunoblastic T-cell lymphoma, age-related immunosenescence may be partly associated with the development of methotrexateassociated T-cell lymphoproliferative disorder and appearance of Epstein-Barr virus-infected cells. On the other hand, Epstein-Barr virus ${ }^{+} \mathrm{B}$ cells could be detected very early in the disease course of angioimmunoblastic T-cell lymphoma. This suggests that Epstein-Barr virus reactivation is not only an event secondary to the associated immune dysfunction, but may also play a role in the development of angioimmunoblastic T-cell lymphoma. Dunleavy et al. [27, 28]. hypothesized that Epstein-Barr virus may play a key role in the early pathogenesis of angioimmunoblastic T-cell lymphoma by activating follicular helper T cells. Thus, EpsteinBarr virus ${ }^{+} \mathrm{B}$ cells may also play a pivotal role in the pathogenesis of methotrexate-associated T-cell lymphoproliferative disorder of angioimmunoblastic T-cell lymphoma type. Considering that the methotrexate-associated T-cell lymphoproliferative disorder of angioimmunoblastic T-cell lymphoma type tended to have scattered Epstein-Barr virus + cells in the background more frequently $(p=0.064)$ than the angioimmuoblastic T-cell lymphoma, it is speculated that this tumorigenic model may contribute more to the pathogenesis of methotrexate-associated T-cell lymphoproliferative disorder of angioimmunoblastic T-cell lymphoma type. Moreover, this model could explain the high frequency of the angioimmunoblastic T-cell lymphoma type in methotrexate-associated T-cell lymphoproliferative disorder.

In the two cases of Epstein-Barr virus ${ }^{-} \mathrm{CD}^{+}$cytotoxic T-cell lymphoma type, scattered Epstein-Barr virus ${ }^{+} \mathrm{B}$ cells were detected in the background. The proliferation of $\mathrm{CD} 8^{+}$ cytotoxic T-cell lymphoma cells may be triggered by the response to the growth of Epstein-Barr virus-infected B cells. $\mathrm{CD}^{+} \mathrm{T}$ cells act to prevent the transformation of Epstein-Barr virus-infected B cells into B-cell malignancy [29]. Similar clonal $\mathrm{CD}^{+}$T-cell expansions has been reported with infectious mononucleosis [30]. Based on the clinical and pathological findings, we are sure that the present $\mathrm{CD} 8^{+}$cytotoxic T-cell lymphoma cases should be diagnosed as lymphoproliferative disorder lesions. However, although speculative, Epstein-Barr virus- $\mathrm{CD} 8^{+}$ cytotoxic T-cell lymphoma type may have the characteristics of a reactive lesion. These characteristics may also account, at least in part, for the extremely favorable clinical behavior of CD8+ cytotoxic T-cell lymphoma type after methotrexate cessation. Very recently, Snadhu et al. [31]. reported that methotrexate preferentially affects subsets of CD8 $+\mathrm{T}$ lymphocytes. They revealed that, on treatment with methotrexate, there was a significant decline in CD8 + IFN $\gamma+$ and increase in CD8 + IL $17+\mathrm{T}$ cells. The potency of methotrexate to increase a subset of $\mathrm{CD} 8+\mathrm{T}$ cells might contribute to the development of CD8+ cytotoxic T-cell lymphoma type.

In summary, methotrexate-associated T-cell lymphoproliferative disorder was divided into three main types: angioimmunoblastic T-cell lymphoma, peripheral T-cell lymphoma, NOS, and $\mathrm{CD}^{+}$cytotoxic T-cell lymphoma. Angioimmunoblastic T-cell lymphoma was the most 
common type. Methotrexate-associated T-cell lymphoproliferative disorder, particularly the $\mathrm{CD} 8^{+}$cytotoxic $\mathrm{T}$-cell lymphoma type, was characterized by a high rate of spontaneous regression after withdrawal of methotrexate. Therefore, methotrexate cessation should be considered as initial management after the diagnosis of methotrexateassociated T-cell lymphoproliferative disorder. Epstein-Barr virus positivity was relatively rare in methotrexateassociated T-cell lymphoproliferative disorder, significantly less frequent than methotrexate-associated B-cell lymphoproliferative disorder, suggesting different pathogenesis. Further analysis in larger series will increase our understanding of methotrexate-associated T-cell lymphoproliferative disorder.

Acknowledgements This work was supported in part by grants from the Grants-in-Aid for Scientific Research, grant number 18K15104.

\section{Compliance with ethical standards}

Conflict of interest The authors declare that they have no conflict of interest.

Publisher's note: Springer Nature remains neutral with regard to jurisdictional claims in published maps and institutional affiliations.

\section{References}

1. Rath T, Rubbert A. Drug combinations with methotrexate to treat rheumatoid arthritis. Clin Exp Rheumatol. 2010;28:S52-7.

2. Bernatsky S, Clarke AE, Suissa S. Hematologic malignant neoplasms after drug exposure in rheumatoid arthritis. Arch Intern Med. 2008;168:378-81.

3. Gaulard P, Swerdlow SH, Harris NL, et al. Other iatrogenic immunodeficiency-associated lymphoproliferative disorders. In: Swerdlow SH, Campo E, Harris NL, et al. editors. World Health Organization classification of tumours of hematopoietic and lymphoid tissue. Revised 4th edn. Lyon, France: International Agency of Research on Cancer; 2017. p. 462-4.

4. Thomas E, Brewster DH, Black RJ, Macfarlane GJ. Risk of malignancy among patients with rheumatic conditions. Int $\mathbf{J}$ Cancer. 2000;88:497-502.

5. Mellemkjaer L, Linet MS, Gridley G, et al. Rheumatoid arthritis and cancer risk. Eur J Cancer. 1996;32a:1753-7.

6. Gridley G, McLaughlin JK, Ekbom A, et al. Incidence of cancer among patients with rheumatoid arthritis. J Natl Cancer Inst. 1993;85:307-11

7. Hoshida Y, Xu JX, Fujita S, et al. Lymphoproliferative disorders in rheumatoid arthritis: clinicopathological analysis of 76 cases in relation to methotrexate medication. J Rheumatol. 2007;34:322-31.

8. Ichikawa A, Arakawa F, Kiyasu J, et al. Methotrexate/iatrogenic lymphoproliferative disorders in rheumatoid arthritis: histology, Epstein-Barr virus, and clonality are important predictors of disease progression and regression. Eur J Haematol. 2013;91:20-8.

9. Mariette X, Cazals-Hatem D, Warszawki J, et al. Lymphomas in rheumatoid arthritis patients treated with methotrexate: a 3-year prospective study in France. Blood. 2002;99:3909-15.

10. Yamakawa N, Fujimoto M, Kawabata D, et al. A clinical, pathological, and genetic characterization of methotrexate- associated lymphoproliferative disorders. J Rheumatol. 2014; 41:293-9.

11. Gion Y, Iwaki N, Takata K, et al. Clinicopathological analysis of methotrexate-associated lymphoproliferative disorders: Comparison of diffuse large B-cell lymphoma and classical Hodgkin lymphoma types. Cancer Sci. 2017;108:1271-80.

12. Hatanaka K, Nakamura N, Kojima M, et al. Methotrexateassociated lymphoproliferative disorders mimicking angioimmunoblastic T-cell lymphoma. Pathol Res Pract. 2010;206:9-13.

13. Ishibuchi $\mathrm{H}$, Motegi $\mathrm{S}$, Yamanaka $\mathrm{M}$, Amano $\mathrm{H}$, Ishikawa $\mathrm{O}$. Methotrexate-associated lymphoproliferative disorder: Sequential development of angioimmunoblastic T-cell lymphoma-like lymphoproliferation in the lymph nodes and diffuse large B-cell lymphoma in the skin in the same patient. Eur $\mathrm{J}$ Dermatol. 2015;25:361-2.

14. Claudino WM, Gibson B, Tse W, Krem M, Grewal J. Methotrexate-associated primary cutaneous CD30-positive cutaneous T-cell lymphoproliferative disorder: a case illustration and a brief review. Am J Blood Res. 2016;6:1-5.

15. Saleh JZ, Lee LH, Schieke SM, Hosking PR, Hwang ST. Methotrexate-induced CD30(+) T-cell lymphoproliferative disorder of the oral cavity. JAAD Case Rep. 2016;2:354-6.

16. Nemoto Y, Taniguchi A, Kamioka M, et al. Epstein-Barr virusinfected subcutaneous panniculitis-like T-cell lymphoma associated with methotrexate treatment. Int J Hematol. 2010;92:364-8.

17. Koji H, Yazawa T, Nakabayashi K, et al. CD8-positive T-cell lymphoproliferative disorder associated with Epstein-Barr virusinfected B-cells in a rheumatoid arthritis patient under methotrexate treatment. Mod Rheumatol. 2016;26:271-5.

18. Hatachi S, Kunitomi A, Aozasa K, Yagita M. CD8(+) T-cell lymphoproliferative disorder associated with Epstein-Barr virus in a patient with rheumatoid arthritis during methotrexate therapy. Mod Rheumatol. 2010;20:500-5.

19. Takajo I, Umekita K, Ikei Y, Oshima K, Okayama A. Adult T-cell leukemia/lymphoma as a methotrexate-associated lymphoproliferative disorder in a patient with rheumatoid arthritis. Intern Med. 2018;57:2071-5.

20. Tokunaga T, Shimada K, Yamamoto K, et al. Retrospective analysis of prognostic factors for angioimmunoblastic T-cell lymphoma: a multicenter cooperative study in Japan. Blood. 2012;119:2837-43.

21. Asano N, Suzuki R, Kagami Y, et al. Clinicopathologic and prognostic significance of cytotoxic molecule expression in nodal peripheral T-cell lymphoma, unspecified. Am J Surg Pathol. 2005;29:1284-93.

22. van Dongen JJ, Langerak AW, Bruggemann M, et al. Design and standardization of PCR primers and protocols for detection of clonal immunoglobulin and T-cell receptor gene recombinations in suspect lymphoproliferations: report of the BIOMED-2 Concerted Action BMH4-CT98-3936. Leukemia. 2003;17:2257-317.

23. Attygalle AD, Kyriakou C, Dupuis J, et al. Histologic evolution of angioimmunoblastic T-cell lymphoma in consecutive biopsies: clinical correlation and insights into natural history and disease progression. Am J Surg Pathol. 2007;31:1077-88.

24. Willenbrock K, Brauninger A, Hansmann ML. Frequent occurrence of B-cell lymphomas in angioimmunoblastic T-cell lymphoma and proliferation of Epstein-Barr virus-infected cells in early cases. Br J Haematol. 2007;138:733-9.

25. Feng WH, Cohen JI, Fischer S, et al. Reactivation of latent Epstein-Barr virus by methotrexate: a potential contributor to methotrexate-associated lymphomas. J Natl Cancer Inst. 2004;96:1691-702.

26. Landais E, Saulquin X, Houssaint E. The human T cell immune response to Epstein-Barr virus. Int J Dev Biol. 2005;49:285-92. 
27. Dunleavy K, Wilson WH. Angioimmunoblastic T-cell lymphoma: immune modulation as a therapeutic strategy. Leuk Lymphoma. 2007;48:449-51.

28. Dunleavy K, Wilson WH, Jaffe ES. Angioimmunoblastic T cell lymphoma: pathobiological insights and clinical implications. Curr Opin Hematol. 2007;14:348-53.

29. Masucci MG, Ernberg I. Epstein-Barr virus: adaptation to a life within the immune system. Trends Microbiol. 1994;2:125-30.
30. Callan MF, Steven N, Krausa P, et al. Large clonal expansions of $\mathrm{CD} 8+\mathrm{T}$ cells in acute infectious mononucleosis. Nat Med. 1996;2:906-11.

31. Sandhu A, Ahmad S, Kaur P, et al. Methotrexate preferentially affects Tc1 and Tc17 subset of CD8 T lymphocytes. Clinical Rheumatol. 2019;38:37-44.

\section{Affiliations}

\section{Akira Satou $^{1} \cdot$ Tetsuya Tabata $^{2} \cdot$ Hiroaki Miyoshi $\mathbb{D}^{3} \cdot$ Kei Kohno $^{4} \cdot$ Yuka Suzuki $^{4} \cdot$ Daisuke Yamashita $^{5}$. Kazuyuki Shimada $^{6} \cdot$ Tomonori Kawasaki $^{7} \cdot$ Yasuharu Sato $^{8} \cdot$ Tadashi Yoshino $^{2} \cdot$ Koichi Ohshima $^{3} \cdot$ Taishi Takahara $^{1}$. Toyonori Tsuzuki ${ }^{1}{ }^{1} \cdot$ Shigeo Nakamura ${ }^{4}$}

1 Department of Surgical Pathology, Aichi Medical University Hospital, Nagakute, Japan

2 Department of Pathology, Dentistry and Pharmaceutical Sciences, Okayama University Graduate School of Medicine,

Okayama, Japan

3 Department of Pathology, School of Medicine, Kurume University, Kurume, Japan

4 Department of Pathology and Laboratory Medicine, Nagoya University Hospital, Nagoya, Japan
5 Department of Pathology, Kobe City Hospital Organization, Kobe City Medical Center General Hospital, Kobe, Japan

6 Department of Hematology and Oncology, Nagoya University Graduate School of Medicine, Nagoya, Japan

7 Department of Pathology, Saitama Medical University International Medical Center, Saitama, Japan

8 Division of Pathophysiology, Okayama University Graduate School of Health Sciences, Okayama, Japan 